\title{
Utilização de medicamentos na gravidez: Riscos e benefícios
}

\author{
Medication use in pregnancy: Risks and benefits
}

Maria Isabelly Brilhante de Aguiar ${ }^{1}$, Jayne Maria Freitas Alves ${ }^{2}$, Joelson Pinheiro

de Lima ${ }^{3}$, Karla Bruna Nogueira Torres ${ }^{4}$

\section{RESUMO}

Durante a gestação a mulher e seu bebê são expostos a riscos, dentre eles, aqueles decorrentes do uso de medicamentos. A utilização dos mesmos nesse período pode ocorrer de maneira inadequada, sendo um problema no âmbito da saúde pública. $A$ pesquisa objetivou verificar a utilização de medicamentos pelas pacientes grávidas atendidas na atenção básica do município de Capistrano, Ceará, bem como o conhecimento destas quanto aos riscos teratogênicos da utilização de fármacos na gravidez. O estudo foi do tipo observacional, descritivo, transversal, sendo um levantamento situacional com abordagem predominantemente quantitativa, desenvolvido no período de setembro a outubro de 2019, com dados obtidos através de entrevistas individuais, que possibilitaram o preenchimento de um formulário semiestruturado. Participaram da pesquisa 50 gestantes, onde $88 \%$ utilizavam medicamentos, sendo mencionados 11 tipos diferentes, pertencentes as classes A e B de risco teratogênico. $62 \%$ das gestantes já tinham recebido alguma orientação a respeito do uso de medicamentos, e apenas $52 \%$ tinham real conhecimento sobre os riscos. É indispensável uma maior educação em saúde permanente dentro das UBS, aumentando o vínculo profissional-paciente, além da inclusão do farmacêutico na equipe multidisciplinar, promovendo o uso racional de medicamentos e diminuindo os riscos de possíveis complicações durante esse período.

Palavras-chave: Gestantes. Teratogênios. Uso de medicamentos.

\section{ABSTRACT}

During pregnancy the woman and her baby are exposed to risks, among them, those arising from the use of medications. Their use in this period can occur inappropriately, being a problem in the field of public health. The research aimed to verify the use of medications by pregnant patients seen in primary care in the city of Capistrano, Ceará, as well as their knowledge regarding the teratogenic risks of the use of drugs in pregnancy. The study was observational, descriptive, transversal, being a situational survey with a predominantly quantitative approach, developed from September to October 2019, with data obtained through individual interviews, which made it possible to fill in a semi-structured form. Fifty pregnant women participated in the research, where $88 \%$ used medicines, with 11 different types being mentioned, belonging to classes $A$ and $B$ of teratogenic risk. $62 \%$ of pregnant women had already received some guidance regarding the use of medications, and only $52 \%$ had real knowledge about the risks. Further education in permanent health within the UBS is essential, increasing the professional-patient bond, in addition to the inclusion of the pharmacist in the multidisciplinary team, promoting the rational use of medications and reducing the risks of possible complications during this period.

Keywords: Pregnant women. Teratogens. Drug utilization. 


\section{INTRODUÇAOO}

A gestação e suas características biológicas, tornam a mulher e seu bebê expostos a riscos, dentre eles, aqueles decorrentes do uso de medicamentos. Por muitos aspectos, tais como idade da paciente, idade gestacional da exposição ao medicamento, fármaco ou substância utilizada, frequência e dose total, é imprescindível uma adequada seleção de medicamentos para evitar riscos, reações e efeitos adversos a mãe e ao feto (MENEZES et al., 2014).

O uso de medicamentos durante a gestação e seus potenciais efeitos sobre o feto em desenvolvimento passou a ser objeto de grande preocupação por estudos de teratogenicidade após a tragédia envolvendo a talidomida. Efeitos colaterais apareceram em mais de 10 mil crianças, após suas mães, nos meses iniciais de gestação usarem a droga para alívio de náuseas e ansiedade (AZEVEDO, 2019).

Os efeitos dos fármacos no feto podem ocorrer em qualquer momento da gravidez, por isso é muito importante conhecer os riscos que eles podem gerar, como más formações e morte causada por medicamentos a serem utilizados, pois grande parte dos fármacos que entram pela circulação materna passando através da placenta, podem produzir efeitos teratogênicos ao feto (NICARRETA et al., 2016).

Apesar dos riscos, o uso de medicamentos durante a gestação é um evento comum para tratar doenças crônicas que a paciente já possuía antes da gravidez, a exemplo da hipertensão, diabetes e depressão, que também podem surgir durante este período, ou manifestações clínicas inerentes à própria gravidez, como enjoos (SANTOS; ALENCAR; ALENCAR, 2013).

Há também casos em que medicamentos, como vitaminas, ferro e ácido fólico, são usados para fins profiláticos na gestação, sendo prescritos com o objetivo de diminuir a probabilidade do efeito teratogênico, além de melhorar o estado de saúde da gestante e do feto (LUNARDI-MAIA; SCHUELTER-TREVISOL; GALATO, 2014).

Devido aos efeitos tóxicos dos fármacos durante a gravidez, o Food And Drug Administration (FDA), um órgão americano, baseado em estudos em animais e humanos, estabeleceu classificações aos medicamentos a partir do seu potencial de risco de danos ao feto durante a gestação. A partir disso, eles foram categorizados nas classes $A, B, C$, $\mathrm{D}$ e $\mathrm{X}$ de risco teratogênico, e essa classificação passou a ser colocada nas bulas (ANDRADE, 2019). 
A relação risco-benefício deve prevalecer na decisão do prescritor em tratar ou não uma gestante. Para uma tomada de decisão sobre uma terapia medicamentosa é sempre aconselhável consultar a literatura especializada, o que poderá ser feito diretamente às fontes de consulta ou através dos centros de informação em medicamentos tanto nas bases nacionais como internacionais (ANDRADE, 2017).

A utilização de medicamentos durante a gravidez é um grande e real problema no âmbito da saúde pública, sendo uma prática muito frequente e na maioria das vezes de maneira inadequada, associado à automedicação para amenizar sintomas comuns do período de gestação ou para tratamento de doenças crônicas ou intercorrentes. Essa prática deve ser evitada ou realizada com muita cautela, uma vez que pode trazer riscos ao feto em desenvolvimento (SANTOS et al., 2018).

Destaca-se que a maior parte dos potenciais efeitos teratogênicos podem ser evitados com o uso racional de medicamentos, o que ressalta a relevância de se conhecer a respeito do uso do mesmos na gestação, colaborando desse modo na identificação da relação risco-benefício do uso desses medicamentos, corrigindo os fatores que contribuem para essa utilização errônea (NAGAI, 2017).

Tendo em vista a perspectiva de que existe uma carência na procura de orientação médica e farmacêutica sobre a utilização de medicamentos na gravidez, surgiu o interesse em obter informações sobre a utilização, benefícios, e possíveis efeitos teratogênicos que o feto está exposto com o uso de medicamentos durante a gestação.

Diante disso, a presente pesquisa teve como objetivo verificar a utilização de medicamentos pelas pacientes grávidas atendidas na atenção básica do município de Capistrano, Ceará, bem como o conhecimento das pacientes quanto aos riscos teratogênicos da utilização de fármacos na gravidez.

\section{MATERIAIS E METODOS}

Realizou-se uma pesquisa de campo do tipo observacional, descritiva, transversal, em forma de um levantamento situacional com abordagem predominantemente quantitativa, em um Centro de Saúde e em três Unidades Básicas de Saúde (UBS) do município de Capistrano, Ceará, estando essas últimas situadas nas comunidades de Boqueirão, Rua videlina e Carqueija. A coleta de dados foi feita nos meses de setembro e outubro de 2019.

A pesquisa foi composta por cinquenta (50) gestantes que eram atendidas nas referidas Unidades Básicas de Saúde e no Centro de Saúde do citado município, no 
período em que a pesquisa foi realizada. Para compor da pesquisa, foram obedecidos os requisitos de inclusão de que participariam gestantes no município de Capistrano-CE, e as mesmas teriam que estar cadastradas e serem atendidas no Centro de Saúde ou em uma das Unidades Básicas de Saúde do município no período do estudo. Foram excluídas as gestantes que não estiveram aptas para argumentar as informações contidas no formulário e que não concordaram em participar da pesquisa, além das gestantes que não estiveram presentes durante a aplicação do questionário.

A análise foi realizada por meio de entrevistas individuais com gestantes que estiveram na fila de espera para a consulta de pré-natal nas Unidades Básicas de Saúde em estudo. Primeiramente foram informadas sobre o trabalho e, em seguida, convidadas para participar da pesquisa. Aquelas que aceitaram participar da pesquisa assinaram 0 Termo de Consentimento Livre e Esclarecido (TCLE).

Os dados foram obtidos a partir do preenchimento do formulário confeccionado para a entrevista, o qual apresentava dezessete perguntas semiestruturadas, que abordaram: o perfil sociodemográfico e gravídico das gestantes (idade, período gestacional, escolaridade, ocupação, número de filhos e de gestação); os sintomas e complicações que a paciente apresentou durante a gestação, dados sobre os medicamentos utilizados (nome, motivo do uso e fontes de orientações adquiridas); o conhecimento dos possíveis riscos que esses fármacos podem oferecer durante a gestação, além da existência de esclarecimento dos mesmos por meio de profissionais da área da saúde capacitados.

Após as transcrições dos dados, iniciou-se a organização do material para a sua análise. A compilação dos dados foi executada no programa Microsoft Office Exce绍 versão 2016 a partir da tabulação das informações obtidas. Em seguida, os dados foram expressos em tabelas.

O presente projeto foi submetido e aprovado pelo Comitê de Ética em Pesquisa do Centro Universitário Católica de Quixadá, através da plataforma Brasil com seu parecer $\mathrm{n}^{\circ}$ 3.257.265, de acordo com os padrões éticos exigidos pela Comissão Nacional de Ética em Pesquisa/Conselho Nacional de Saúde/Ministério da Saúde (CONEP/ CNS/MS), conforme Resolução 466/12, que regulamenta as diretrizes e normas das pesquisas com seres humanos. 


\section{RESULTADOS E DISCUSSAOO}

A presente pesquisa contou com a participação de 50 gestantes cadastradas em três UBS e em um Centro de saúde do município de Capistrano, Ceará, com idades entre 15 a 38 anos, sendo que 34\% das gestantes tinham idades entre 22 a 27 anos.

No aspecto escolaridade, a maioria das grávidas tinham ensino médio completo (52\%) seguido do fundamental incompleto (14\%) e superior completo (14\%). Quanto a ocupação verificou-se que $48 \%$ eram agricultoras e $26 \%$ donas de casa. Condições que podem influenciar diretamente ou indiretamente nos resultados obtidos nas consultas de pré-natal.

Tabela 1- Descrição do perfil sociodemográfico e gravídico das gestantes participantes do estudo, Capistrano- Ceará - Brasil, 2019.

\begin{tabular}{lcc|lcr}
\hline \multicolumn{5}{c}{ Tabela 1 - PERFIL SOCIODEMOGRÁFICO E GRAVÍDICO DAS GESTANTES } \\
\hline $\begin{array}{l}\text { PERFIL } \\
\text { SOCIODEMOGRÁFICO }\end{array}$ & N & $\%$ & PERFIL GRAVÍDICO & N & $\%$ \\
\hline Idade (anos) & & & Período gestacional (mês) & & \\
\hline $15-20$ & 15 & 30 & $1-3$ & 10 & 20 \\
\hline $22-27$ & 17 & 34 & $4-6$ & 25 & 50 \\
\hline $28-32$ & 08 & 16 & $7-9$ & 15 & 30 \\
\hline $33-38$ & 10 & 20 & Número de gestação & & \\
\hline Escolaridade & & & 01 & 26 & 52 \\
\hline Ensino Fundamental incompleto & 07 & 14 & 02 & 13 & 26 \\
\hline Ensino Fundamental completo & 05 & 10 & 03 & 09 & 18 \\
\hline Ensino Médio incompleto & 05 & 10 & 04 & 02 & 04 \\
\hline Ensino Médio completo & 26 & 52 & Número de filhos & 26 & 52 \\
\hline Ensino Superior completo & 07 & 14 & 00 & 14 & 28 \\
\hline Ocupação & & & 01 & 08 & 16 \\
\hline Agricultora & 24 & 48 & 02 & 02 & 04 \\
\hline Dona de casa & 13 & 26 & 03 & 34 & 68 \\
\hline Professora & 06 & 12 & Planejamento da gestação & 16 & 32 \\
\hline Estudante & 03 & 06 & Foi planejada & & \\
\hline Outros & 04 & 08 & Não foi planejada & & \\
\hline & & Fonte: & Autores. & & \\
\hline
\end{tabular}

Os dados apresentados anteriormente corroboram com um estudo realizado no Vale do Taquari, Rio Grande do Sul, em que 25 gestantes participantes apresentaram uma faixa etária entre 15 a 37 anos, tendo mais da metade delas concluído o ensino médio (NICARRETA et al., 2016).

Relacionando esses dados com o consumo de medicamentos na gravidez, foi observado que ocorre um aumento da utilização de medicamentos quando as gestantes 
possuem uma escolaridade mais elevada, pois há uma maior procura do cuidado da saúde, através dos serviços prestados pelo Sistema Único de Saúde (SUS), bem como um maior acesso aos medicamentos (BORGES et al., 2017).

A pesquisa mostrou que metade das gestantes se encontravam no segundo trimestre de gestação, sendo que a maioria delas (52\%) estavam na sua primeira gestação e não possuíam filhos. O fato de parte das gestantes estarem em sua primeira gestação não justifica, porém esclarece a falta de algumas informações a respeito do uso de medicamentos nesse período, já que não é de conhecimento de muitas das mesmas a capacidade que alguns fármacos têm de passar pela placenta e chegar ao feto.

Considerando a variável planejamento da gravidez, grande parte das gestantes (68\%) declararam que a gravidez foi planejada, o que pode auxiliar em um uso de medicamentos de forma mais controlada, devido muitas mulheres buscarem informações em geral a respeito dos cuidados que se deve tomar antes e durante esse período quando há um planejamento.

Alguns dados citados se assemelham com pesquisa realizada onde a metade das mulheres do estudo não teve nenhuma gestação anterior, mostrando dúvidas a respeito do uso de medicamentos, e se difere em relação ao planejamento da gravidez, onde nessa pesquisa $61,1 \%$ relataram não ter planejado a mesma (COSTA; COELHO; SANTOS, 2017).

Entre as 50 gestantes entrevistadas 8 delas relataram ter problemas de saúde, tendo destaque para asma, com $50 \%$ dos casos, e hipertensão, com $37,5 \%$ de citações, sendo que algumas gestantes relataram ter mais de um problema. Das gestantes participantes, 31 tiveram complicações gestacionais, com a prevalência de anemia $(51,6 \%)$ seguida de infecção urinaria $(32,3 \%)$. Essas complicações e problemas de saúde que surgiram antes ou durante a gestação, são fatores desencadeadores do uso de medicamentos, ocorrendo a prescrição medicamentosa na tentativa de além de tratar as complicações, reduzir as chances de uma possível gestação de risco, que pode levar a uma morte materna e fetal.

Tabela 2 - Problemas de saúde e complicações gestacionais relatados pelas gestantes participantes do estudo, Capistrano- Ceará - Brasil, 2019.

\begin{tabular}{|c|c|c|c|c|c|}
\hline Problemas de saúde & $\mathbf{N}$ & $\%$ & Complicacões & $\mathbf{N}$ & $\%$ \\
\hline Asma & 04 & 50 & Anemia & 16 & 51,6 \\
\hline
\end{tabular}




\begin{tabular}{llll|lcc} 
& Hipertensão & 03 & 37,5 & Infecção urinaria & 10 & 32,3 \\
\hline Diabetes & 01 & 12,5 & Sangramento & 03 & 9,7 \\
\cline { 2 - 6 } Fonte: & 01 & 12,5 & Coluna e circulação & 01 & 3,2 \\
\cline { 2 - 5 } Rinite & 01 & 12,5 & Eclampsia & 01 & 3,2 \\
\cline { 2 - 6 }
\end{tabular}

A asma é uma comorbidade com potenciais impactos graves sobre gestação. Seu mau controle poderá causar hipoxemia, hipocapnia e alcalose materna, afetando à circulação materno-fetal, resultando em risco de aborto espontâneo, prematuridade, síndromes hipertensivas na gestação, hiperêmese gravídica e baixo peso neonatal (COSTA, 2017).

A Hipertensão arterial é uma doença cardiovascular com condição clínica multifatorial, identificada pela alteração nos níveis de pressão arterial $\geq 140$ / $90 \mathrm{mmHg}$, atingindo cerca de 5 a $10 \%$ das gestantes, derivada do aumento da resistência vascular periférica, podendo resultar em altos riscos para a saúde da mãe e do feto se não for controlada (RODRIGUES et al., 2018).

Durante a gestação é muito comum haver uma carência de ferro, devido a um amento da sua demanda para suprir tanto a mãe quanto o feto, pois cerca de $300 \mathrm{mg}$ ou mais de ferro do corpo da mãe são transportados para o feto e para a placenta, podendo ocasionar complicações, dentre elas quadros anêmicos maternos e fetais, que se desfazem após o parto (ANDRADE et al., 2017).

A infecção do trato urinário também é uma complicação muito frequente entre as gestantes, devido às várias mudanças fisiológicas que ocorrem durante a gravidez, gerando uma vulnerabilidade ao organismo da mulher quanto ao aumento de cepas microbianas, facilitando, assim, o desenvolvimento das infecções. Essa complicação pode desenvolver ruptura prematura de membranas amnióticas, restrição de crescimento intraútero, um parto prematuro, recém-nascidos de baixo peso e até mesmo a morte do feto (CARVALHO, 2015; RAMOS et al., 2016).

Em relação a utilização de medicamentos, 44 (88\%) grávidas utilizavam medicamentos e apenas 6 (12\%) optaram por não fazer o uso dos mesmos durante a gestação. Foram mencionados 11 tipos diferentes de medicamentos, entre eles 04 (36\%) eram da classe A (FDA) e 07 (64\%) eram da classe B (FDA) de risco teratogênico, não foram mencionados medicamentos das classes $C, D$ e $X$ (FDA). $O$ sulfato ferroso foi o medicamento mais mencionado, com $24(34,2 \%)$ de todas as citações, ficando o ácido fólico logo em seguida, com $23(33 \%)$, os dois da classe de risco $A$, seguido do paracetamol e cefalexina, ambos do risco B, compondo $7,2 \%$ cada um entre os 
medicamentos utilizados, ressaltando que muitas gestantes utilizavam mais de um medicamento.

Tabela 3 - Medicamentos utilizados pelas gestantes participantes do estudo, Capistrano - Ceará Brasil, 2019.

Tabela 3 - MEDICAMENTOS UTILIZADOS PELAS GESTANTES

\begin{tabular}{|c|c|c|c|c|c|c|c|c|c|c|}
\hline \multirow[t]{3}{*}{ Medicamentos } & \multirow[t]{3}{*}{ FDA } & \multirow[t]{3}{*}{$\begin{array}{l}\text { Motivo do } \\
\text { uso }\end{array}$} & \multirow{2}{*}{\multicolumn{3}{|c|}{$\begin{array}{c}\text { Com prescrição } \\
\text { médica/ } \\
\text { enfermeira/ } \\
\text { farmacêutica }\end{array}$}} & \multicolumn{3}{|c|}{$\begin{array}{c}\text { Sem } \\
\text { prescrição }\end{array}$} & \multirow{2}{*}{\multicolumn{2}{|c|}{$\begin{array}{c}\text { Total de } \\
\text { medicamentos } \\
\text { utilizados }\end{array}$}} \\
\hline & & & & & & & mes & & & \\
\hline & & & $1^{\circ}$ & $2^{\circ}$ & $3^{\circ}$ & $1^{\circ}$ & $2^{\circ}$ & $3^{\circ}$ & $\mathbf{N}$ & $\%$ \\
\hline Ácido Fólico & A & $\begin{array}{l}\text { Formação do } \\
\text { feto }\end{array}$ & 13 & 06 & 03 & 01 & 00 & 00 & 23 & 33 \\
\hline $\begin{array}{l}\text { Sulfato } \\
\text { Ferroso }\end{array}$ & A & Anemia & 09 & 05 & 09 & 00 & 01 & 00 & 24 & 34,2 \\
\hline Materna & A & vitamina & 00 & 00 & 00 & 00 & 01 & 00 & 01 & 1,4 \\
\hline Dactil-OB & A & Sangramento & 01 & 00 & 00 & 00 & 00 & 00 & 01 & 1,4 \\
\hline Metildopa & B & $\begin{array}{c}\text { Picos } \\
\text { hipertensivos }\end{array}$ & 01 & 01 & 00 & 00 & 00 & 00 & 02 & 2,8 \\
\hline Cefalexina & B & $\begin{array}{l}\text { Infecção } \\
\text { urinaria }\end{array}$ & 02 & 02 & 00 & 00 & 01 & 00 & 05 & 7,2 \\
\hline Nitrofurantoina & B & $\begin{array}{l}\text { Infecção } \\
\text { urinaria }\end{array}$ & 00 & 01 & 00 & 00 & 00 & 00 & 01 & 1,4 \\
\hline Insulina & B & Diabetes & 01 & 00 & 00 & 00 & 00 & 00 & 01 & 1,4 \\
\hline Loratadina & B & Rinite & 00 & 01 & 00 & 00 & 00 & 00 & 01 & 1,4 \\
\hline Prednisona & B & Asma & 01 & 01 & 00 & 00 & 00 & 00 & 02 & 2,8 \\
\hline Paracetamol & B & $\begin{array}{c}\text { Febre e dores } \\
\text { de cabeça }\end{array}$ & 02 & 01 & 01 & 00 & 01 & 00 & 05 & 7,2 \\
\hline Não lembra & - & Não lembra & 02 & 01 & 01 & 00 & 00 & 00 & 04 & 5,8 \\
\hline
\end{tabular}

É de grande importância o uso da suplementação vitamínica e mineral durante a gestação, o ácido fólico e sulfato ferroso são usados como parte de protocolos ou recomendações para o período gestacional. Durante a primeira consulta pré-natal os profissionais da atenção básica prescrevem ácido fólico, com intuito de prevenir as anormalidades congênitas, além de reduzir as chances de prematuridade, ruptura precoce da placenta e do baixo peso do feto ao nascer (COSTA; COELHO; SANTOS, 2017).

Embora o Ministério da Saúde recomende em seus protocolos o uso de sulfato ferroso na gestação devido sua contribuição na prevenção de anemias nesse período, seu uso generalizado iniciou um questionamento se a suplementação profilática de ferro seria necessária ou deveria ser utilizada apenas quando a anemia fosse diagnosticada (COSTA; COELHO; SANTOS, 2017). 
Durante a gravidez, a cefalexina na dose de $500 \mathrm{mg}$ é o medicamento da classe das cefalosporinas mais utilizado, pois atinge um nível superior em combate contra os coliformes e tem uma baixa toxicidade em casos de uso em gestantes e correlação ao feto (SILVA; SOUSA; VITORINO, 2019).

O paracetamol é um AINE que ativa vias e receptores envolvidos na produção de dor, podendo inibir a síntese de prostaglandina central e modulando as vias inibitórias serotoninérgicas descendentes. É considerado um analgésico seguro nas doses recomendadas de acordo com a literatura para o tratamento da dor durante a gestação. Porém, em controversa, alguns estudos relatam a possibilidade do uso de paracetamol durante a gravidez estar associado ao aumento dos riscos de desenvolvimento de algumas síndromes, como o autismo e transtorno de hiperatividade, todavia, ainda necessita de estudos mais detalhados para ser comprovado (ANDRADE et al., 2018).

Vale ressaltar que apesar do risco do uso de medicamentos utilizados pelas gestantes não estar estabelecido, é importante a diminuição de sua utilização, ainda que estejam em classificação de segurança, tendo em vista os riscos que podem causar devido essa população ser limitada a estudos com medicamentos na fase de desenvolvimento embrionário.

Esses resultados se assemelham com uma pesquisa realizado no Hospital Instituto Cândida Vargas, em que 79,34\% das grávidas faziam uso de algum fármaco e apenas $20,66 \%$ das gestantes disseram que não ingeriram nenhuma medicação, sendo o ácido fólico e sulfato ferroso os mais utilizados (DANTAS et al., 2018).

No entanto, a pesquisa citada se diferencia quanto à relação de risco teratogênico, uma vez que na de Dantas et al. (2018), dos medicamentos citados, 59,5\% pertenciam à classe de risco $A, 33,88 \%$ à classe de risco $B, 2,48 \%$ à classe de risco $C$ e $4,14 \%$ à classe de risco D. Já na presente investigação houve a preponderância de fármacos pertencentes à classe de risco $\mathrm{B}$, com $64 \%$ das citações, seguida de $36 \%$ da classe de risco A. Vale ressaltar que em ambos os estudos não foi detectado o uso de medicamentos de Risco X.

Os motivos mais relatados do uso de medicação durante a gestação pelas gestantes foram prevenção à anemia e ajuda na formação do feto, tratamento de infecção urinaria e dores. Salienta-se que 5,8\% das gestantes entrevistadas não lembravam o medicamento e motivo do uso dos mesmos.

Foi verificado que $33(47,1 \%)$ medicamentos utilizados, foram por gestantes que se encontravam no primeiro trimestre de gestação. Sendo um resultado preocupante, tendo 
em vista que é o período da organogênese e o de maior risco de efeitos teratogênicos, porém a ida aos pré-natais desde os primeiros meses de gestação influenciou o uso de medicamentos nesse período. O que corrobora com a pesquisa feita por Nicaretta et al. (2016) na qual foi observado um grande percentual de uso dos medicamentos $(43,7 \%)$ durante o primeiro trimestre da gestação.

Com relação à prescrição dos medicamentos na gestação, $46 \%$ das gestantes relataram ter sido prescritos por um médico, 41\% disseram ter sido prescrito por uma enfermeira da UBS, e apenas $2 \%$ relatou ter sido indicado por um farmacêutico. $11 \%$ das gestantes expuseram ter usado medicamento por indicação de outras pessoas que não são profissionais da saúde, como vizinhos e familiares. Tais resultados se assemelham a aos estudos de Nascimento et al. (2016), em que $80 \%$ das gestantes responderam não administrar medicamentos sem prescrição médica, bem como com a pesquisa de Costa, Coelho e Santos (2017) na qual mais da metade das gestantes utilizavam medicamentos com prescrição.

Foi observado na presente pesquisa que 31 (62\%) das gestantes receberam orientações sobre o uso de medicamentos na gestação, e 19 (38\%) não receberam. Foi possível observar também que apenas 26 (52\%) das gestantes tinham real conhecimento sobre os riscos do uso de medicamento na gravidez e 24 (48\%), não tinham esse conhecimento. Muitas das gestantes relataram que receberam informações dos médicos e enfermeiros das UBS sobre os riscos e benefícios do uso, porém acabaram esquecendo. Outras já disseram que obtiveram informações pela internet e por vizinhos.

Tabela 4 - Orientações e o conhecimento sobre a utilização de medicamentos pelas gestantes participantes do estudo, Capistrano - Ceará - Brasil, 2019.

\begin{tabular}{|c|c|c|}
\hline Variáveis & $\mathbf{N}$ & $\%$ \\
\hline \multicolumn{3}{|c|}{ Recebeu alguma orientação } \\
\hline Sim & 31 & 62 \\
\hline Não & 19 & 38 \\
\hline \multicolumn{3}{|c|}{ Conhecimento das gestantes sobre os riscos } \\
\hline Tinha conhecimento & 26 & 52 \\
\hline Não tinha conhecimento & 24 & 48 \\
\hline
\end{tabular}

Fonte: Autores.

Em outra pesquisa foi observado que a maioria das gestantes entrevistadas também tinham sido devidamente informadas sobre os riscos do uso de medicamentos na 
gravidez, e que $88 \%$ das mesmas tinham algum conhecimento sobre o risco do uso de medicamentos durante a gestação. (NICARETTA et al., 2016).

Isso revela que, atualmente, as gestantes estão cada vez mais buscando informações sobre os possíveis riscos que podem estar expostas com a utilização de medicamentos, sendo um perigo a procura dessas informações em fontes não confiáveis, como pode acontecer internet por exemplo, tornando esse repasse de conhecimento adquirido através de profissionais da saúde ainda mais necessário.

\section{CONSIDERAÇOES FINAIS}

Pode-se concluir que o uso de medicamentos na gestação merece especial atenção da equipe multidisciplinar das UBS, tendo em vista que muitas gestantes estavam fazendo uso dos mesmos, principalmente no período da organogênese. Notou-se também que apesar das gestantes estarem bem informadas e os medicamentos utilizados não serem potencialmente de risco, é importante que se tenha uma maior educação em saúde permanente dentro da UBS, com o uso materiais e métodos educativos sobre o uso racional de medicamentos principalmente na gravidez, aumentando 0 vínculo profissional/paciente. Ademais, é fundamental a ampla divulgação de informações pela equipe multiprofissional para que as dúvidas relacionadas aos medicamentos sejam supridas.

Diante disso, julga-se importante a inclusão do farmacêutico na equipe multidisciplinar das UBS, tendo em vista que esse profissional é o detentor do conhecimento sobre a farmacoterapia. Além de agregar ações de auxílio de efetividade na adesão de tratamentos, contribuindo na avaliação do risco-benefício e segurança em relação ao potencial teratogênico, realizando ações de educação e orientação da terapia medicamentosa, promovendo o uso racional de medicamentos e diminuindo, dessa forma, os riscos de possíveis complicações durante a gestação.

\section{REFERÉNCIAS}

ANDRADE, Andréia Moreira et al. Farmacocinética e mecanismos de teratogenicidade dos medicamentos na gestação: uma revisão da literatura. Infarma - Ciências Farmacêuticas, v. 29, n. 2, p. 100-107, 2017.

ANDRADE, Andréia Moreira de et al. Utilização de anti-inflamatórios, analgésicos e antipiréticos na gestação: uma revisão narrativa. Journal of Biology \& Pharmacy and Agricultural Management, v. 14, n. 2, p. 87-97, abr./jun. 2018. 
ANDRADE, Andréia Moreira de. Utilização de medicamentos em gestantes no município de Rio Branco, Acre. 2017. 182 f. Tese (Doutorado em Ciências) - Escola Nacional de Saúde Pública Sergio Arouca, Fundação Oswaldo Cruz, Rio de Janeiro, 2017.

ANDRADE, Tamires Oliveira. Influência do consumo de álcool, tabaco e medicamentos durante o período gestacional e avaliação dos riscos teratogênicos. 2019. 65 f. Monografia (Graduação em Farmácia) - Escola de farmácia, Universidade Federal de Ouro Preto, Ouro Preto, 2019.

AZEVEDO, Aline Abrahão. O uso de medicamentos durante a gestação e o papel da farmacovigilância. 2019. 32 f. Monografia (Especialização em Tecnologia Industriais Farmacêuticas) - Instituto de Tecnologia em Fármacos, Fundação Oswaldo Cruz, Rio de Janeiro, 2019.

BORGES, Vinícius Miranda et al. Uso de medicamentos entre gestantes de um município no sul de Minas Gerais, Brasil. Infarma - Ciências Farmacêuticas, v. 30, n. 1, p. 30-43, 2017.

CARVALHO, Clebner Inácio de. Infecção do trato urinário associado a gestantes e o papel do profissional farmacêutico no tratamento farmacoterapêutico. FACIDER Revista Científica, Colider, n. 7, 2015.

COSTA, Débora Bomfim; COELHO, Helena Lutescia Luna; SANTOS, Djanilson Barbosa dos. Utilização de medicamentos antes e durante a gestação: prevalência e fatores associados. Cad. Saúde Pública, v. 33, n. 2, 2017.

COSTA, Eric de Medeiros. Asma e gravidez: uma revisão de literatura. 2017. 46 f. Monografia (Graduação em Medicina) - Centro de Ciências Biológicas e da Saúde, Universidade Federal do Maranhão, São Luís, 2017.

DANTAS, Allana Carla et al. Avaliação de medicamentos por gestantes em hospital de referência. Revista da Universidade Vale do Rio Verde, v. 16, n. 2, ago./dez. 2018.

LUNARDI-MAIA, Tânia; SCHUELTER-TREVISOL, Fabiana; GALATO, Dayani. Uso de medicamentos no primeiro trimestre de gravidez: avaliação da segurança dos medicamentos e uso de ácido fólico e sulfato ferroso. Rev Bras Ginecol Obstet., v. 36, n. 12, p. 541-7, 2014.

MENEZES, Marília Stefani Souza de et al. Uso de medicamentos por gestantes atendidas no Hospital da Polícia Militar - Mossoró/RN. Rev. Bras. Farm., v. 95, n. 1, p. 512-529, 2014.

NAGAI, Michelly Martins. Utilização de medicamentos por gestantes de alto risco no Hospital da Clínicas da Faculdade de Medicina de Ribeirão Preto da Universidade de São Paulo - HCFMRP-USP. 2017. 198 f. Tese (Doutorado em Área de concentração: Medicamentos e Cosméticos) - Faculdade de Ciências Farmacêuticas de Ribeirão, Universidade de São Paulo, Ribeirão Preto, 2017. 
NASCIMENTO, Áurea Miranda do et al. Avaliação do uso de medicamentos por gestantes em unidades básicas de saúde de Rondonópolis, Mato Grosso. Revista Eletrônica Gestão \& Saúde, v. 7, n. 1, p. 96-12, 2016.

NICARETTA, Fernanda Micaela Richter et al. Utilização de medicamentos por gestantes usuárias do sistema único de saúde em um município do Vale do Taquari - RS.

Destaques Acadêmicos, Lajeado, v. 8, n. 3, p. 7-19, 2016.

RAMOS, Géssica Capellin et al. Prevalência de infecção do trato urinário em gestantes em uma cidade no Sul do Brasil. Saúde (Santa Maria), v. 42, n. 1, p. 173-178, jan./jun. 2016.

RODRIGUES, Aline de Souza et al. Assistência farmacêutica no âmbito de cuidados a gestantes com hipertensão arterial. Rev Cient FAEMA, Ariquemes, v. 9, n. ed esp, p. 540-546, maio./jun. 2018.

SANTOS, Paloma Oliveira dos; ALENCAR, Tatiane de Oliveira Silva; ALENCAR, Bruno Rodrigues. Medicamentos e gravidez: uma análise dos estudos de utilização de medicamentos realizados no brasil (2000 - 2011). Revista Eletrônica Gestão \& Saúde, v. 4, n. 3, p. 1102-15, 2013.

SANTOS, Sandna Larissa Freitas dos et al. Automedicação em gestantes de alto risco: foco em atenção farmacêutica. Rev Med UFC., v. 58, n. 3, p. 36-43, 2018.

SILVA, Raimunda de Abreu; SOUSA, Thainara Araújo de; VITORINO, Keila de Assis. Infecção do trato urinário na gestação: diagnóstico e tratamento. Rev Cient FAEMA, Ariquemes, v. 10, n. 1, p. 71-80, jan./jun. 2019. 